\title{
On subclass of meromorphic multivalent functions associated with Liu-Srivastava operator
}

\author{
Saqib Hussain ${ }^{\mathrm{a}}$, Jamila Bibi ${ }^{\mathrm{a}}$, Mohsan Raza ${ }^{\mathrm{b}, *}$, Maslina Darus ${ }^{\mathrm{c}}$ \\ ${ }^{a}$ COMSATS Institute of Information Technology, Abbotabad, Pakistan. \\ ${ }^{b}$ Department of Mathematics, Government College University Faisalabad, Pakistan. \\ ${ }^{c}$ School of Mathematical Sciences, Faculty of Science and Technology, Universiti Kebangsaan Malaysia 43600, Bangi, Selangor, \\ Malaysia.
}

Communicated by S.-H. Rim

\begin{abstract}
In the present paper, we introduce a new subclass related to meromorphically p-valent reciprocal starlike functions associated with the Liu-Srivastava operator. Some sufficient conditions for functions belonging to this class are derived. The results presented here improve and generalize some known results. (C)2017 All rights reserved.
\end{abstract}

Keywords: Meromorphic functions, convolution, linear operator.

2010 MSC: 30C45, 30C50.

\section{Introduction}

Let $\Sigma_{p}$ denote the class of meromorphic functions of the form

$$
f(z)=z^{-p}+\sum_{k=1}^{\infty} a_{k} z^{k-p}, \quad(p \in \mathbb{N}=\{1,2, \cdots\}),
$$

which are analytic and p-valent in the punctured open unit disc $\mathbb{U}^{*}=\{z \in \mathbb{C}: 0<|z|<1\}=\mathbb{U} \backslash\{0\}$, where $\mathbb{U}$ is the open unit disk $\mathbb{U}=\{z \in \mathbb{C}:|z|<1\}$. In particular, we set $\Sigma_{1}=\Sigma$. Let $f$ and $g$ be two analytic functions in the open unit disk $\mathbb{U}$, we say that the function $f$ is subordinate to $g$ (written as $f \prec g$ ) if there exists a Schwarz function $\omega$, which is analytic in $\mathbb{U}$ with $\omega(0)=0$ and $|\omega(z)|<1$, such that $f(z)=g(\omega(z))$. Furthermore, if the function $g$ is univalent in $\mathbb{U}$, then we have the following equivalent relation:

$$
f(z) \prec g(z) \Longleftrightarrow f(0)=g(0) \quad \text { and } \quad f(\mathbb{U}) \subset g(\mathbb{U}) .
$$

\footnotetext{
*Corresponding author

Email addresses: saqib_math@yahoo.com (Saqib Hussain), syyedajameela@gmail.com (Jamila Bibi), mohsan976@yahoo.com (Mohsan Raza), maslina@ukm.edu.my (Maslina Darus)

doi:10.22436/jnsa.010.09.35
} 
For some details see [2, 12]; see also [15].

A function $f \in \Sigma_{p}$ is said to be in class $\mathcal{S}_{\mathfrak{p}}^{*}(\oslash)$ of meromorphically p-valent starlike of order $\alpha$ if and only if

$$
\Re e\left(\frac{z f^{\prime}(z)}{\operatorname{pf}(z)}\right)<-\alpha, \quad(0 \leqslant \alpha<1) .
$$

It is clear that $\mathcal{S}_{\mathrm{p}}^{*}(0)=\mathcal{S}_{\mathrm{p}}^{*}$, the class of $\mathrm{p}$-valent starlike functions. A function $f \in \mathcal{S}_{\mathrm{p}}^{*}$ is said to be in the class $\mathcal{M}_{p}(\oslash)$ of meromorphically p-valent starlike of reciprocal order $\alpha$ if and only if

$$
\Re e\left(\frac{p f(z)}{z f^{\prime}(z)}\right)<-\alpha, \quad(0 \leqslant \alpha<1) .
$$

In particular $\mathcal{M}_{1}(\oslash)=\mathcal{M}(\oslash)$.

Remark 1.1. In view of the fact that

$$
\mathfrak{R e}(\mathrm{p}(z))<0 \Longrightarrow \mathfrak{R e}\left(\frac{1}{\mathrm{p}(z)}\right)=\mathfrak{R e}\left(\frac{\mathrm{p}(z)}{|\mathrm{p}(z)|^{2}}\right)<0,
$$

it follows that meromorphically p-valent starlike function of reciprocal order 0 is same as a meromorphically p-valent starlike function. When $0<\alpha<1$, the function $f \in \Sigma_{p}$ is meromorphically $p$-valent starlike of reciprocal order $\alpha$ if and only if

$$
\left|\frac{z f^{\prime}(z)}{\operatorname{pf}(z)}+\frac{1}{2 \alpha}\right|<\frac{1}{2 \alpha}
$$

For $p=1$, this class was studied by Sun et al. [17]. For arbitrary fixed real numbers $A$ and $B(-1 \leqslant$ $B<A \leqslant 1)$, we denote by $P(A, B)$ the class of the functions of the form

$$
\mathrm{q}(z)=1+\mathrm{c}_{1} z+\mathrm{c}_{2} z^{2}+\cdots,
$$

which is analytic in the unit disk $\mathbb{U}$ and satisfies the condition

$$
\mathrm{q}(z) \prec \frac{1+\mathrm{Az}}{1+\mathrm{B} z}, \quad(z \in \mathbb{U}) .
$$

The class $P(A, B)$ was introduced and studied by Janowski [5]. We also observe from (1.2) (see also [14]) that a function $q(z) \in P(A, B)$ if and only if

$$
\left|q(z)-\frac{1-A B}{1-B^{2}}\right|<\frac{A-B}{1-B^{2}}, \quad(B \neq-1),
$$

and

$$
\mathfrak{R e}\{\mathbf{q}(z)\}>\frac{1-\mathrm{A}}{2}, \quad(\mathrm{~B}=-1) .
$$

For function $f \in \Sigma_{p}$ given by (1.1) and $g \in \Sigma_{p}$ given by

$$
g(z)=z^{-p}+\sum_{k=1}^{\infty} b_{k} z^{k-p},
$$

the Hadamard product (convolution) of $f$ and $g$ is given by

$$
(\mathrm{f} * \mathrm{~g})(z)=z^{-p}+\sum_{k=1}^{\infty} a_{k} b_{k} z^{k-p}=(g * f)(z) .
$$

For complex parameters $\alpha_{i}$ and $\beta_{j}$, where $i=1,2, \cdots, l, j=1,2, \cdots, m$ and $\beta_{j} \notin \mathbb{Z}_{\mathrm{o}}^{-}=\{0,-1,-2, \cdots\}$, the generalized hypergeometric function ${ }_{l} \mathcal{F}_{m}$ is defined by

$$
{ }_{l} \mathcal{F}_{m}\left(\alpha_{1}, \ldots, \alpha_{l} ; \beta_{1}, \ldots, \beta_{m}\right)(z)=\sum_{k=0}^{\infty} \frac{\left(\alpha_{1}\right)_{k} \cdots\left(\alpha_{l}\right)_{k}}{k !\left(\beta_{1}\right)_{k} \cdots\left(\beta_{m}\right)_{k}} z^{k},
$$

where $l \leqslant m+1, l, m \in \mathbb{N}_{0}=\{0,1,2, \ldots\}$ and $(\lambda)_{\mathfrak{n}}$ is pochhammer symbol (or shifted factorial) defined 
in terms of Gamma function by

$$
(\lambda)_{n}=\frac{\Gamma(\lambda+n)}{\Gamma(\lambda)}=\left\{\begin{array}{lr}
1, & n=0, \\
\lambda(\lambda+1) \cdots(\lambda+n-1), & n \in \mathbb{N} .
\end{array}\right.
$$

Now consider the function

$$
h_{p}\left(\alpha_{1}, \ldots, \alpha_{l} ; \beta_{1}, \ldots, \beta_{m} ; z\right)=z^{-p_{l} \mathcal{F}_{m}}\left(\alpha_{1}, \ldots, \alpha_{l} ; \beta_{1}, \ldots, \beta_{m}\right)(z),
$$

then the Liu-Srivastava linear operator $[8,9] \mathcal{H}_{p}\left(\alpha_{1}, \ldots, \alpha_{l} ; \beta_{1}, \ldots, \beta_{m}\right): \Sigma_{p} \longrightarrow \Sigma_{p}$ is defined by using the Hadamard product (or convolution) as

$$
\begin{aligned}
\mathcal{H}_{p}\left(\alpha_{1}, \ldots, \alpha_{l} ; \beta_{1}, \ldots, \beta_{m}\right) f(z) & =h_{p}\left(\alpha_{1}, \ldots, \alpha_{l} ; \beta_{1}, \ldots, \beta_{m} ; z\right) * f(z) \\
& =z^{-p}+\sum_{k=1}^{\infty} \frac{\left(\alpha_{1}\right)_{k} \cdots\left(\alpha_{l}\right)_{k}}{k !\left(\beta_{1}\right)_{k} \cdots\left(\beta_{m}\right)_{k}} a_{k} z^{k-p} .
\end{aligned}
$$

For convenience, we denote $\mathcal{H}_{p}\left(\alpha_{1}, \ldots, \alpha_{l} ; \beta_{1}, \ldots, \beta_{m}\right) \approx \mathcal{H}_{p, l, m}\left[\alpha_{1}\right]$.

The Liu-Srivastava operator is studied in $[1,13,16]$, is the meromorphic analogue of the DziokSrivastava [3] linear operator. Special cases of the Liu-Srivastava linear operator include the meromorphic analogue of the Carlson-Shaffer linear operator $L_{p}(a, c)=\mathcal{H}_{p, 2,1}(1, a, c)$ studied among others by Liu and Srivastava [7], Liu [6] and Yang [20]. The analogous to the Ruscheweyh derivative operator $\mathrm{D}^{\mathrm{n}+1}=\mathrm{L}_{\mathrm{p}}(\mathrm{n}+\mathrm{p}, 1)$ was investigated by Yang [19]. The operator

$$
\mathrm{J}_{\mathrm{c}, \mathrm{p}}=\frac{\mathrm{c}}{z^{\mathrm{c}+\mathrm{p}}} \int_{0}^{z} \mathrm{t}^{\mathrm{c}+\mathrm{p}-1} \mathrm{f}(\mathrm{t}) \mathrm{dt}=\mathrm{L}_{\mathrm{p}}(\mathrm{c}, \mathrm{c}+1), \quad(\mathrm{c}>0),
$$

was studied by Uralegaddi and Somanatha [18].

By using operator $\mathcal{H}_{p, l, m}\left[\alpha_{1}\right]$, we introduce the following new class.

Definition 1.2. A function $f \in \Sigma_{p}$ is said to be in the class $\mathcal{M}_{\left[\alpha_{1}\right]}\left(p ; \beta ; \lambda ; A_{1}, B\right)$, if it satisfies the subordination

$$
\frac{p}{1-p \beta}\left\{\frac{(1-\lambda) \mathcal{H}_{p, l, m}\left[\alpha_{1}\right] f(z)+\lambda z\left(\mathcal{H}_{p, l, m}\left[\alpha_{1}\right] f(z)\right)^{\prime}}{z\left(\mathcal{H}_{p, l, m}\left[\alpha_{1}\right] f(z)\right)^{\prime}+\lambda z^{2}\left(\mathcal{H}_{p, l, m}\left[\alpha_{1}\right] f(z)\right)^{\prime \prime}}+\beta\right\} \prec-\frac{1+A_{1} z}{1+B z},
$$

where $A_{1}=(1-\alpha) A+\alpha B, 0 \leqslant \alpha<1,0 \leqslant \lambda \leqslant 1,-1 \leqslant B<A \leqslant 1,0 \leqslant p \beta<1$ and $\mathcal{H}_{p, l, m}\left[\alpha_{1}\right]$ is defined in (1.5).

Remark 1.3. Using (1.3), (1.4) and for $B \neq-1$, the Definition 1.2 is equivalent to

$$
\left|\frac{p}{1-p \beta}\left\{\frac{\mathcal{H}_{p, l, m}\left[\alpha_{1}\right] F_{\lambda}(z)}{z\left(\mathcal{H}_{p, l, m}\left[\alpha_{1}\right] F_{\lambda}(z)\right)^{\prime}}+\beta\right\}+\frac{1-A_{1} B}{1-B^{2}}\right|<\frac{A_{1}-B}{1-B^{2}},
$$

and for $\mathrm{B}=-1$,

$$
\mathfrak{R e}\left\{\frac{p}{1-p \beta}\left\{\frac{\mathcal{H}_{p, l, m}\left[\alpha_{1}\right] \mathrm{F}_{\lambda}(z)}{z\left(\mathcal{H}_{\mathrm{p}, \mathrm{l}, \mathrm{m}}\left[\alpha_{1}\right] \mathrm{F}_{\lambda}(z)\right)^{\prime}}+\beta\right\}\right\}<-\frac{1-\mathrm{A}_{1}}{2}
$$

also, for $B=-1, A_{1} \neq 1,(1.7)$ reduces to

$$
\left|\frac{1-p \beta}{p}\left(\frac{z\left(\mathcal{H}_{p, l, m}\left[\alpha_{1}\right] F_{\lambda}(z)\right)^{\prime}}{\mathcal{H}_{p, l, m}\left[\alpha_{1}\right] F_{\lambda}(z)+\beta z\left(\mathcal{H}_{p, l, m}\left[\alpha_{1}\right] F_{\lambda}(z)\right)^{\prime}}\right)+\frac{1}{1-A_{1}}\right|<\frac{1}{1-A_{1}},
$$

and for $B=-1, A_{1}=1$, we obtain

$$
\left|\frac{p}{1-p \beta}\left(\frac{\mathcal{H}_{p, l, m}\left[\alpha_{1}\right] F_{\lambda}(z)}{z\left(\mathcal{H}_{p, l, m}\left[\alpha_{1}\right] F_{\lambda}(z)\right)^{\prime}}+\beta\right)+1\right|<1,
$$

where

$$
F_{\lambda}(z)=(1-\lambda) f(z)+\lambda z f^{\prime}(z) .
$$


By assigning particular values to parameters the class $\mathcal{M}_{\left[\alpha_{1}\right]}(p ; \alpha ; \beta ; \lambda ; A, B)$ generalizes many previously known classes of meromorphic functions.

(i) For $\lambda=0, \alpha=0, l=2, m=1, \alpha_{1}=a, \alpha_{2}=1, \beta_{1}=c$, the class $\mathcal{M}_{\left[\alpha_{1}\right]}\left(p ; \beta ; \lambda ; A_{1}, B\right)$ coincides with the class studied in [10].

(ii) For $p=1, A=1-2 \gamma, 0<\gamma<1, \beta=0, B=-1, a=c=1$, the class $\mathcal{M}_{\left[\alpha_{1}\right]}\left(p ; \beta ; \lambda ; A_{1}\right.$, B) coincides with the class studied in [17].

\section{Preliminaries}

We need the following lemmas for our future investigation.

Lemma 2.1 (Jack's lemma [4]). Let the (non constant) function $\omega(z)$ be analytic in $\mathbb{U}$, with $\omega(0)=0$. If $|\omega(z)|$ attains its maximum value on the circle $|z|=r<1$ at a point $z_{0} \in \mathbb{U}$, then $z_{0} \omega^{\prime}\left(z_{0}\right)=\gamma \omega\left(z_{0}\right)$, where $\gamma$ is real number and $\gamma \geqslant 1$.

Lemma 2.2 ([11]). Let $\Omega$ be a set in the complex plane $\mathbb{C}$ and suppose that $\phi$ is a complex mapping from $\mathbb{C}^{2} \times \mathbb{U}$ to $\mathbb{C}$ which satisfies $\phi(i x, y ; z) \notin \Omega$ for $z \in \mathbb{U}$, and for all real $x, y$ such that $y \leqslant-\frac{1+x^{2}}{2}$. If the function $p(z)=1+c_{1} z+c_{2} z^{2}+\cdots$ is analytic in $\mathbb{U}$ and $\phi\left(p(z), z p^{\prime}(z) ; z\right) \in \Omega$ for all $z \in \mathbb{U}$, then $\operatorname{Re}(p(z))>0$.

Lemma 2.3 ([20]). Let $\mathrm{p}(z)=1+\mathrm{b}_{1} z+\mathrm{b}_{2} z^{2}+\cdots$, be analytic in $\mathbb{U}$ and $\eta$ be analytic and starlike (with respect to the origin) univalent in $\mathbb{U}$ with $\eta(0)=0$. If $z p^{\prime}(z) \prec \eta(z)$ then

$$
p(z) \prec 1+\int_{0}^{z} \frac{\eta(t)}{t} d t .
$$

Unless otherwise mentioned, we shall assume that $0 \leqslant \alpha<1,0 \leqslant \lambda \leqslant 1,-1 \leqslant B<A \leqslant 1,0 \leqslant p \beta<1$ and $p \in \mathbb{N}$.

\section{Main results}

Theorem 3.1. Let $\mathrm{f} \in \Sigma_{\mathrm{p}}$. Then $\mathrm{f} \in \mathcal{M}_{\left[\alpha_{1}\right]}(\mathrm{p} ; \alpha ; \beta ; \lambda ; A, B)$ if and only if

$$
\frac{p}{1-p \beta}\left\{\frac{\mathcal{H}_{p, l, m}\left[\alpha_{1}\right] F_{\lambda}(z)}{z\left(\mathcal{H}_{p, l, m}\left[\alpha_{1}\right] F_{\lambda}(z)\right)^{\prime}}+\beta\right\} \prec-\frac{1+A_{1} z}{1+B z}
$$

Proof. If $f \in \mathcal{M}_{\left[\alpha_{1}\right]}(p ; \alpha ; \beta ; \lambda ; A, B)$, then

$$
\frac{p}{1-p \beta}\left\{\frac{(1-\lambda) \mathcal{H}_{p, l, m}\left[\alpha_{1}\right] f(z)+\lambda z\left(\mathcal{H}_{p, l, m}\left[\alpha_{1}\right] f(z)\right)^{\prime}}{z\left(\mathcal{H}_{p, l, m}\left[\alpha_{1}\right] f(z)\right)^{\prime}+\lambda z^{2}\left(\mathcal{H}_{p, l, m}\left[\alpha_{1}\right] f(z)\right)^{\prime \prime}}+\beta\right\} \prec-\frac{1+A_{1} z}{1+B z} .
$$

Let

$$
F_{\lambda}(z)=(1-\lambda) f(z)+\lambda z f^{\prime}(z)
$$

so

$$
\mathcal{H}_{\mathrm{p}, \mathrm{l}, \mathrm{m}}\left[\alpha_{1}\right] \mathrm{F}_{\lambda}(z)=(1-\lambda) \mathcal{H}_{\mathrm{p}, \mathrm{l}, \mathrm{m}}\left[\alpha_{1}\right] \mathrm{f}(z)+\lambda z \mathcal{H}_{\mathrm{p}, \mathrm{l}, \mathrm{m}}\left[\alpha_{1}\right] \mathrm{f}^{\prime}(z) .
$$

Using (3.2), (3.3) and after some simplifications we have

$$
\frac{p}{1-p \beta}\left\{\frac{\mathcal{H}_{p, l, m}\left[\alpha_{1}\right] F_{\lambda}(z)}{z\left(\mathcal{H}_{p, l, m}\left[\alpha_{1}\right] F_{\lambda}(z)\right)^{\prime}}+\beta\right\} \prec-\frac{1+A_{1} z}{1+B z},
$$

the converse is straight forward. 
Theorem 3.2. If $\mathrm{f} \in \Sigma_{\mathrm{p}}$ satisfies anyone of the following conditions

(i) for $\mathrm{B} \neq-1$

$$
\begin{aligned}
& \sum_{k=1}^{\infty}\left(\begin{array}{c}
\left|(k-p) \lambda_{1}\right|+ \\
\quad
\end{array} \quad \begin{array}{c}
\left|p\left(1-B^{2}\right)\left[(1+(k-p) \beta) \lambda_{1}\right]+\left(1-A_{1} B\right)(1-p \beta)(k-p) \lambda_{1}\right| \\
(1-p \beta)\left(\left|A_{1}\right|-B\right)
\end{array}\right)\left|\Gamma_{k}\left(\alpha_{1}\right)\right|\left|a_{k}\right| \\
& \quad<p|1-\lambda-\lambda p|(1-|B|) ;
\end{aligned}
$$

(ii) for $\mathrm{B}=-1, \mathrm{~A}_{1} \neq 1$

$$
\begin{aligned}
& \sum_{k=1}^{\infty}\left(\begin{array}{c}
\left|(1+(k-p) \beta) \lambda_{1}\right|+ \\
\quad<(1-p \beta)\left(1-\left|A_{1}\right|\right)|1-\lambda-\lambda p|
\end{array}\right)\left|\Gamma_{k}\left(\alpha_{1}\right)\right|\left|a_{k}\right| \\
& \quad(k-p) \beta) \lambda_{1}+\frac{\left.\left(1-A_{1}\right)(1-p \beta)(k-p) \lambda_{1}\right)}{p} \mid
\end{aligned}
$$

(iii) for $\mathrm{B}=-1, \mathrm{~A}_{1}=1$

$$
\sum_{k=1}^{\infty}\left(\left|(k-p) \lambda_{1}\right|+\frac{k\left|\lambda_{1}\right|}{1-p \beta}\right)\left|\Gamma_{k}\left(\alpha_{1}\right)\right|\left|a_{k}\right|<p\left|\lambda_{1}\right|,
$$

then $f \in \mathcal{M}_{\left[\alpha_{1}\right]}(p ; \alpha ; \beta ; \lambda ; A, B)$, where $\lambda_{1}=1+\lambda(k-p-1)$ with $\Gamma_{k}\left(\alpha_{1}\right)=\frac{\left(\alpha_{1}\right)_{k} \cdots\left(\alpha_{l}\right)_{k}}{k !\left(\beta_{1}\right)_{k} \cdots\left(\beta_{m}\right)_{k}}$.

Proof. (i): If $B \neq-1$, by the condition (1.6) we only need to show that

$$
\left|\frac{p\left(1-B^{2}\right)}{(1-p \beta)\left(A_{1}-B\right)}\left\{\frac{\mathcal{H}_{p, l, m}\left[\alpha_{1}\right] F_{\lambda}(z)}{z\left(\mathcal{H}_{p, l, m}\left[\alpha_{1}\right] F_{\lambda}(z)\right)^{\prime}}+\beta\right\}+\frac{1-A_{1} B}{A_{1}-B}\right|<1 .
$$

We first observe the

$$
\begin{aligned}
& \left|\frac{p\left(1-B^{2}\right)}{(1-p \beta)\left(A_{1}-B\right)}\left\{\frac{\mathcal{H}_{p, l, m}\left[\alpha_{1}\right] F_{\lambda}(z)}{z\left(\mathcal{H}_{p, l, m}\left[\alpha_{1}\right] F_{\lambda}(z)\right)^{\prime}}+\beta\right\}+\frac{1-A_{1} B}{A_{1}-B}\right| \\
& \quad=\left|\frac{p B(1-\lambda-\lambda p)+\sum_{k=1}^{\infty} \frac{p\left(1-B^{2}\right)\left[(1+(k-p) \beta) \lambda_{1}\right]+\left(1-A_{1} B\right)(1-p \beta)(k-p) \lambda_{1}}{(1-p \beta)\left(\left|A_{1}\right|-B\right)} \Gamma_{k}\left(\alpha_{1}\right) a_{k} z^{k} \mid}{-p(1-\lambda-\lambda p)+\sum_{k=1}^{\infty}(k-p) \lambda_{1} \Gamma_{k}\left(\alpha_{1}\right) a_{k} z^{k}}\right| \\
& \leqslant \frac{p|B(1-\lambda-\lambda p)|+\sum_{k=1}^{\infty} \frac{\left|p\left(1-B^{2}\right)\left[(1+(k-p) \beta) \lambda_{1}\right]+\left(1-A_{1} B\right)(1-p \beta)(k-p) \lambda_{1}\right|}{(1-p \beta)\left(\left|A_{1}\right|-B\right)}\left|\Gamma_{k}\left(\alpha_{1}\right)\right|\left|a_{k}\right||z|^{k}}{p|1-\lambda-\lambda p|-\sum_{k=1}^{\infty}\left|(k-p) \lambda_{1}\right|\left|\Gamma_{k}\left(\alpha_{1}\right)\right|\left|a_{k}\right||z|^{k}} \\
& <\frac{p|B(1-\lambda-\lambda p)|+\sum_{k=1}^{\infty} \frac{\left|p\left(1-B^{2}\right)\left[(1+(k-p) \beta) \lambda_{1}\right]+\left(1-A_{1} B\right)(1-p \beta)(k-p) \lambda_{1}\right|}{(1-p \beta)\left(\left|A_{1}\right|-B\right)}\left|\Gamma_{k}\left(\alpha_{1}\right)\right|\left|a_{k}\right|}{p|1-\lambda-\lambda p|-\sum_{k=1}^{\infty}\left|(k-p) \lambda_{1}\right|\left|\Gamma_{k}\left(\alpha_{1}\right)\right|\left|a_{k}\right|} .
\end{aligned}
$$

Now, by using the inequality (3.4), we have

$$
\frac{p|B(1-\lambda-\lambda p)|+\sum_{k=1}^{\infty} \frac{\left|p\left(1-B^{2}\right)\left[(1+(k-p) \beta) \lambda_{1}\right]+\left(1-A_{1} B\right)(1-p \beta)(k-p) \lambda_{1}\right|}{(1-p \beta)\left(\left|A_{1}\right|-B\right)}\left|\Gamma_{k}\left(\alpha_{1}\right)\right|\left|a_{k}\right|}{p|1-\lambda-\lambda p|-\sum_{k=1}^{\infty}\left|(k-p) \lambda_{1}\right|\left|\Gamma_{k}\left(\alpha_{1}\right)\right|\left|a_{k}\right|}<1,
$$

which, in conjunction with (3.7), completes the proof of (i) for Theorem 3.2. 
(ii): If $B=-1, A_{1} \neq 1$, by the virtue of the condition (1.8), we only need to show that

$$
\left|\frac{\left(1-A_{1}\right)(1-p \beta)}{p}\left(\frac{z\left(\mathcal{H}_{p, l, m}\left[\alpha_{1}\right] F_{\lambda}(z)\right)^{\prime}}{\mathcal{H}_{p, l, m}\left[\alpha_{1}\right] F_{\lambda}(z)+\beta z\left(\mathcal{H}_{p, l, m}\left[\alpha_{1}\right] F_{\lambda}(z)\right)^{\prime}}\right)+1\right|<1 .
$$

We first observe that

$$
\begin{aligned}
& \left|\frac{\left(1-A_{1}\right)(1-p \beta)}{p}\left(\frac{z\left(\mathcal{H}_{p, l, m}\left[\alpha_{1}\right] F_{\lambda}(z)\right)^{\prime}}{\mathcal{H}_{p, l, m}\left[\alpha_{1}\right] F_{\lambda}(z)+\beta z\left(\mathcal{H}_{p, l, m}\left[\alpha_{1}\right] F_{\lambda}(z)\right)^{\prime}}\right)+1\right| \\
& =\left|\frac{A_{1}(1-p \beta)(1-\lambda-\lambda p)+\sum_{k=1}^{\infty}\left((1+(k-p) \beta) \lambda_{1}+\frac{\left(1-A_{1}\right)(1-p \beta)(k-p) \lambda_{1}}{p}\right) \Gamma_{k}\left(\alpha_{1}\right) a_{k} z^{k}}{(1-p \beta)(1-\lambda-\lambda p)+\sum_{k=1}^{\infty}(1+(k-p) \beta) \lambda_{1} \Gamma_{k}\left(\alpha_{1}\right) a_{k} z^{k}}\right| \\
& \leqslant \frac{\left|A_{1}\right||1-\lambda-\lambda p|(1-p \beta)+\sum_{k=1}^{\infty}\left(\left|(1+(k-p) \beta) \lambda_{1}+\frac{\left(1-A_{1}\right)(1-p \beta)(k-p) \lambda_{1}}{p}\right|\right)\left|\Gamma_{k}\left(\alpha_{1}\right)\right|\left|a_{k}\right|\left|z^{k}\right|}{(1-p \beta)|(1-\lambda-\lambda p)|-\sum_{k=1}^{\infty}\left|(1+(k-p) \beta) \lambda_{1}\right|\left|\Gamma_{k}\left(\alpha_{1}\right)\right|\left|a_{k}\right|\left|z^{k}\right|} \\
& <\frac{\left|A_{1}\right||1-\lambda-\lambda p|(1-p \beta)+\sum_{k=1}^{\infty}\left(\left|(1+(k-p) \beta) \lambda_{1}+\frac{\left(1-A_{1}\right)(1-p \beta)(k-p) \lambda_{1}}{p}\right|\right)\left|\Gamma_{k}\left(\alpha_{1}\right)\right|\left|a_{k}\right|}{(1-p \beta)|1-\lambda-\lambda p|-\sum_{k=1}^{\infty}\left|(1+(k-p) \beta) \lambda_{1}\right|\left|\Gamma_{k}\left(\alpha_{1}\right)\right|\left|a_{k}\right|} .
\end{aligned}
$$

By using the inequality (3.5), we have

$$
\frac{\left|A_{1}\right||1-\lambda-\lambda p|(1-p \beta)+\sum_{k=1}^{\infty}\left(\left|(1+(k-p) \beta) \lambda_{1}+\frac{\left(1-A_{1}\right)(1-p \beta)(k-p) \lambda_{1}}{p}\right|\right)\left|\Gamma_{k}\left(\alpha_{1}\right)\right|\left|a_{k}\right|}{(1-p \beta)|1-\lambda-\lambda p|-\sum_{k=1}^{\infty}\left|(1+(k-p) \beta) \lambda_{1}\right|\left|\Gamma_{k}\left(\alpha_{1}\right)\right|\left|a_{k}\right|}<1,
$$

which, in conjunction with (3.8), completes the proof of (ii) for Theorem 3.2.

(iii): If $B=1, A_{1}=1$, by virtue of the condition (1.9), we only need to show that

$$
\left|\frac{p}{1-p \beta}\left(\frac{\mathcal{H}_{p, l, m}\left[\alpha_{1}\right] F_{\lambda}(z)}{z\left(\mathcal{H}_{p, l, m}\left[\alpha_{1}\right] F_{\lambda}(z)\right)^{\prime}}+\beta\right)+1\right|<1, \quad(z \in \mathbb{U}) .
$$

We first observe that

$$
\begin{aligned}
\left|\frac{p}{1-p \beta}\left(\frac{\mathcal{H}_{p, l, m}\left[\alpha_{1}\right] F_{\lambda}(z)}{z\left(\mathcal{H}_{p, l, m}\left[\alpha_{1}\right] F_{\lambda}(z)\right)^{\prime}}+\beta\right)+1\right| & =\left|\frac{\sum_{k=1}^{\infty} \frac{k \lambda_{1}}{1-p \beta} \Gamma_{k}\left(\alpha_{1}\right) a_{k} z^{k}}{-p(1-\lambda-\lambda p)+\sum_{k=1}^{\infty}(k-p) \lambda_{1} \Gamma_{k}\left(\alpha_{1}\right) a_{k} z^{k}}\right| \\
& \leqslant \frac{\sum_{k=1}^{\infty} \frac{k\left|\lambda_{1}\right|}{1-p \beta}\left|\Gamma_{k}\left(\alpha_{1}\right)\right|\left|a_{k}\right||z|^{k}}{p|1-\lambda-\lambda p|-\sum_{k=1}^{\infty}\left|(k-p) \lambda_{1}\right|\left|\Gamma_{k}\left(\alpha_{1}\right)\right|\left|a_{k}\right||z|^{k}} \\
& <\frac{\sum_{k=1}^{\infty} \frac{k\left|\lambda_{1}\right|}{1-p \beta}\left|\Gamma_{k}\left(\alpha_{1}\right)\right|\left|a_{k}\right|}{p|1-\lambda-\lambda p|-\sum_{k=1}^{\infty}\left|(k-p) \lambda_{1}\right|\left|\Gamma_{k}\left(\alpha_{1}\right)\right|\left|a_{k}\right|}
\end{aligned}
$$

Now, by using the inequality (3.6), we have

$$
\frac{\sum_{k=1}^{\infty} \frac{k\left|\lambda_{1}\right|}{1-p \beta}\left|\Gamma_{k}\left(\alpha_{1}\right)\right|\left|a_{k}\right|}{p|1-\lambda-\lambda p|-\sum_{k=1}^{\infty}\left|(k-p) \lambda_{1}\right|\left|\Gamma_{k}\left(\alpha_{1}\right)\right|\left|a_{k}\right|}<1,
$$


which, in conjunction with (3.9), completes the proof of (iii), for Theorem 3.2.

Theorem 3.3. If $\mathrm{f} \in \Sigma_{\mathrm{p}}$ satisfies anyone of the following conditions:

(i) for $\mathrm{B} \neq-1$,

$$
\left|L_{p, l, m}^{\alpha_{1}}(F(z))\right|<\frac{(1-p \beta)\left(A_{1}-B\right)}{(1-p \beta)\left(A_{1}-B\right)+(1+|B|)} ;
$$

(ii) for $\mathrm{B}=-1,-1<\mathrm{A}_{1} \leqslant 0$

$$
\left|\mathrm{L}_{\mathrm{p}, \mathrm{l}, \mathrm{m}}^{\alpha_{1}}(\mathrm{~F}(z))\right|<\frac{(1-\mathrm{p} \beta)\left(1-\mathrm{A}_{1}\right)\left(1+\mathrm{A}_{1}\right)}{2 \mathrm{p} \beta\left(1+\mathrm{A}_{1}\right)+2\left(1-\mathrm{A}_{1}\right)}
$$

(iii) for $\mathrm{B}=-1, \mathrm{~A}_{1}=1$

$$
\left|\mathrm{L}_{p, l, m}^{\alpha_{1}}(\mathrm{~F}(z))\right|<\frac{1-p \beta}{2-p \beta}
$$

then $\left.f \in \mathcal{M}_{\left[\alpha_{1}\right]} p ; \alpha ; \beta ; \lambda ; A, B\right)$, where

$$
\mathrm{L}_{\mathrm{p}, \mathrm{l}, \mathrm{m}}^{\alpha_{1}}(\mathrm{~F}(z))=1+\frac{z\left(\mathcal{H}_{\mathrm{p}, \mathrm{l}, \mathrm{m}}\left[\alpha_{1}\right] \mathrm{F}_{\lambda}(z)\right)^{\prime \prime}}{\left(\mathcal{H}_{\mathrm{p}, \mathrm{l}, \mathrm{m}}\left[\alpha_{1}\right] \mathrm{F}_{\lambda}(z)\right)^{\prime}}-\frac{z\left(\mathcal{H}_{\mathrm{p}, \mathrm{l}, \mathrm{m}}\left[\alpha_{1}\right] \mathrm{F}_{\lambda}(z)\right)^{\prime}}{\left(\mathcal{H}_{\mathrm{p}, \mathrm{l}, \mathrm{m}}\left[\alpha_{1}\right] \mathrm{F}_{\lambda}(z)\right)} .
$$

Proof. (i) If $B \neq-1$, let

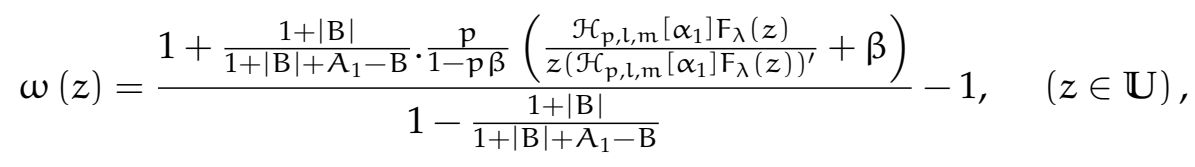

then the function $\omega$ is analytic in $\mathbb{U}$ with $\omega(0)=0$. Using (3.11) and after some simplifications, we obtain

$$
\frac{p \mathcal{H}_{p, l, m}\left[\alpha_{1}\right] F_{\lambda}(z)}{z\left(\mathcal{H}_{p, l, m}\left[\alpha_{1}\right] F_{\lambda}(z)\right)^{\prime}}=\frac{(1-p \beta)\left(A_{1}-B\right) \omega(z)-(1+|B|)}{1+|B|}
$$

Differentiating both sides of (3.12), logarithmically we get

$$
\mathrm{L}_{p, l, m}^{\alpha_{1}}(F(z))=-\frac{(1-p \beta)\left(A_{1}-B\right) z \omega^{\prime}(z)}{(1-p \beta)\left(A_{1}-B\right) \omega(z)-(1+|B|)} .
$$

By virtue of (3.10) and (3.13), we find that

$$
\left|\mathrm{L}_{p, l, m}^{\alpha_{1}}(F(z))\right|=(1-p \beta)\left(A_{1}-B\right)\left|\frac{z \omega^{\prime}(z)}{(1-p \beta)\left(A_{1}-B\right) \omega(z)-(1+|B|)}\right|,
$$

and

$$
\left|L_{p, l, m}^{\alpha_{1}}(F(z))\right|<\frac{(1-p \beta)\left(A_{1}-B\right)}{(1-p \beta)\left(A_{1}-B\right)+(1+|B|)} .
$$

Next, we claim that $|\omega(z)|<1$. Indeed, if not, there exists a point $z_{0} \in \mathbb{U}$ such that

$$
\max _{|z| \leqslant\left|z_{0}\right|}|\omega(z)|=\left|\omega\left(z_{0}\right)\right|=1, \quad\left(z_{0} \in \mathbb{U}\right) .
$$

Applying Lemma 2.1 to $\omega(z)$ at the point $z_{0}$, we have

$$
z_{0} \omega^{\prime}\left(z_{0}\right)=\gamma \omega\left(z_{0}\right), \quad(\gamma \geqslant 1) .
$$


By writing

$$
\omega\left(z_{0}\right)=e^{i \theta}, \quad(0 \leqslant \theta \leqslant 2 \pi),
$$

and setting $z=z_{0}$ in (3.13), we get

$$
\left|L_{p, l, m}^{\alpha_{1}}\left(F\left(z_{0}\right)\right)\right|=(1-p \beta)\left(A_{1}-B\right)\left|\frac{\gamma}{(1-p \beta)\left(A_{1}-B\right)-(1+|B|) e^{-i \theta}}\right|,
$$

which implies

$$
\left|L_{p, l, m}^{\alpha_{1}}\left(F\left(z_{0}\right)\right)\right| \geqslant(1-p \beta)\left(A_{1}-B\right)\left|\frac{1}{(1-p \beta)\left(A_{1}-B\right)-(1+|B|) e^{-i \theta}}\right| .
$$

This implies that

$$
\left|L_{p, l, m}^{\alpha_{1}}(F(z))\right|^{2} \geqslant \frac{\left[(1-p \beta)\left(A_{1}-B\right)\right]^{2}}{\left[(1-p \beta)\left(A_{1}-B\right)\right]^{2}+(1+|B|)^{2}-2(1-p \beta)\left(A_{1}-B\right)(1+|B|) \cos \theta} .
$$

Since the right hand side of (3.14) takes its minimum value for $\cos \theta=-1$, we have

$$
\left|L_{p, l, m}^{\alpha_{1}}\left(F\left(z_{0}\right)\right)\right|^{2} \geqslant \frac{\left[(1-p \beta)\left(A_{1}-B\right)\right]^{2}}{\left[(1-p \beta)\left(A_{1}-B\right)+(1+|B|)\right]^{2}} .
$$

This implies that

$$
\left|\frac{p}{1-p \beta}\left(\frac{\mathcal{H}_{p, l, m}\left[\alpha_{1}\right] F_{\lambda}(z)}{z\left(\mathcal{H}_{p, l, m}\left[\alpha_{1}\right] F_{\lambda}(z)\right)^{\prime}}+\beta\right)+1\right|<\frac{A_{1}-B}{1+|B|}
$$

then, we have

$$
\begin{aligned}
& \left|\frac{p}{1-p \beta}\left(\frac{\mathcal{H}_{p, l, m}\left[\alpha_{1}\right] F_{\lambda}(z)}{z\left(\mathcal{H}_{p, l, m}\left[\alpha_{1}\right] F_{\lambda}(z)\right)^{\prime}}+\beta\right)+\frac{1-A_{1} B}{1-B^{2}}\right| \\
& \quad \leqslant\left|\frac{p}{1-p \beta}\left(\frac{\mathcal{H}_{p, l, m}\left[\alpha_{1}\right] F_{\lambda}(z)}{z\left(\mathcal{H}_{p, l, m}\left[\alpha_{1}\right] F_{\lambda}(z)\right)^{\prime}}+\beta\right)+1\right|+\left|\frac{1-A_{1} B}{1-B^{2}}-1\right| \\
& \quad<\frac{A_{1}-B}{(1+|B|)}+\frac{|B|\left(A_{1}-B\right)}{1-B^{2}} \\
& \quad=\frac{A_{1}-B}{1-B^{2}}, \quad(B \neq-1) .
\end{aligned}
$$

Therefore, we conclude that $f(z) \in \mathcal{M}_{\left[\alpha_{1}\right]}(p ; \alpha ; \beta ; \lambda ; A, B)$ for $B \neq-1$.

Using similar arguments as in proof of (i), (ii) and (iii) can be easily verified.

Theorem 3.4. If $f \in \Sigma_{p}$ satisfies

$$
\mathfrak{R e}\left(L_{p, l, m}^{\alpha_{1}}(F(z))\right)< \begin{cases}\frac{\beta_{2}}{2(1-p \beta)\left(A_{1}-B\right)}, & B+\frac{1-B}{2(1-p \beta)} \leqslant A_{1} \leqslant 1, \\ \frac{(1-p \beta)\left(A_{1}-B\right)}{2 \beta_{2}}, & B<A_{1} \leqslant B+\frac{1-B}{2(1-p \beta)},\end{cases}
$$

then $f(z) \in M_{\left[\alpha_{1}\right]}(p ; \alpha ; \beta ; \lambda ; A, B)$, where $\beta_{2}=\left(1-A_{1}\right)+p \beta\left(A_{1}-B\right)$.

Proof. Let

$$
g(z)=\frac{\frac{-p}{1-p \beta}\left\{\frac{\mathcal{H}_{p, l, m}\left[\alpha_{1}\right] \mathrm{F}_{\lambda}(z)}{z\left(\mathcal{H}_{p, l, m}\left[\alpha_{1}\right] \mathrm{F}_{\lambda}(z)\right)^{\prime}}+\beta\right\}-\frac{1-\mathrm{A}_{1}}{1-\mathrm{B}}}{1-\frac{1-\mathrm{A}_{1}}{1-\mathrm{B}}} .
$$

Then $\mathrm{g}$ is analytic in $\mathbb{U}$. Using (3.16), we have

$$
\frac{-p \mathcal{H}_{p, l, m}\left[\alpha_{1}\right] F_{\lambda}(z)}{z\left(\mathcal{H}_{p, l, m}\left[\alpha_{1}\right] F_{\lambda}(z)\right)^{\prime}}=\frac{(1-p \beta)\left(A_{1}-B\right) g(z)+\beta_{2}}{1-B} .
$$


Differentiating (3.17) logarithmically, we obtain

$$
-L_{p, l, m}^{\alpha_{1}}(F(z))=\frac{(1-p \beta)\left(A_{1}-B\right) z g^{\prime}(z)}{(1-p \beta)\left(A_{1}-B\right) g(z)+\beta_{2}}=\prec\left(g(z), z g^{\prime}(z) ; z\right),
$$

where

$$
\prec(r, s ; t)=\frac{(1-p \beta)\left(A_{1}-B\right) s}{(1-p \beta)\left(A_{1}-B\right) r+\beta_{2}} .
$$

For all real $x$ and $y$ satisfying $y \leqslant-\frac{1+x^{2}}{2}$, we have

$$
\begin{aligned}
\mathfrak{R e}(\prec(i x, y ; z)) & =\frac{(1-p \beta)\left(A_{1}-B\right) \beta_{2} y}{\left(\beta_{2}\right)^{2}+\left[(1-p \beta)\left(A_{1}-B\right)\right]^{2} x^{2}} \\
& \leqslant-\frac{1+x^{2}}{2} \cdot \frac{(1-p \beta)\left(A_{1}-B\right) \beta_{2}}{\left(\beta_{2}\right)^{2}+\left[(1-p \beta)\left(A_{1}-B\right)\right]^{2} x^{2}} \\
& \leqslant \begin{cases}-\frac{\beta_{2}}{2(1-p \beta)\left(A_{1}-B\right)}, & B+\frac{1-B}{2(1-p \beta)} \leqslant A_{1} \leqslant 1, \\
-\frac{(1-p \beta)\left(A_{1}-B\right)}{2 \beta_{2}}, & B<A_{1} \leqslant B+\frac{1-B}{2(1-p \beta)} .\end{cases}
\end{aligned}
$$

We know put

$$
\Omega=\left\{\xi: \mathfrak{R e}(\xi)>\left\{\begin{array}{ll}
-\frac{\beta_{2}}{2(1-p \beta)\left(A_{1}-B\right)}, & B+\frac{1-B}{2(1-p \beta)} \leqslant A_{1} \leqslant 1, \\
-\frac{(1-p \beta)\left(A_{1}-B\right)}{2 \beta_{2}}, & B<A_{1} \leqslant B+\frac{1-B}{2(1-p \beta)},
\end{array}\right\},\right.
$$

then $\prec(i x, y ; z) \notin \Omega$ for all real $x, y$ such that $y \leqslant-\frac{1+x^{2}}{2}$. Moreover, in view of (3.15), we know that $\prec\left(g(z), z g^{\prime}(z) ; z\right) \in \Omega$. Thus by Lemma 2.2 we deduce that

$$
\mathfrak{R e}(\mathrm{g}(z))>0, \quad(z \in \mathbb{U}),
$$

which shows that the desired assertion of Theorem 3.4 holds.

Theorem 3.5. If $f \in \Sigma_{p}$ satisfies

$$
\mathfrak{R e}\left\{\frac{\mathrm{p} \mathcal{H}_{\mathrm{p}, \mathrm{l}, \mathrm{m}}\left[\alpha_{1}\right] \mathrm{F}_{\lambda}(z)}{z\left(\mathcal{H}_{\mathrm{p}, \mathrm{l}, \mathrm{m}}\left[\alpha_{1}\right] \mathrm{F}_{\lambda}(z)\right)^{\prime}}\left(1+\eta \frac{z\left(\mathcal{H}_{\mathrm{p}, \mathrm{l}, \mathrm{m}}\left[\alpha_{1}\right] \mathrm{F}_{\lambda}(z)\right)^{\prime \prime}}{\left(\mathcal{H}_{\mathrm{p}, \mathrm{l}, \mathrm{m}}\left[\alpha_{1}\right] \mathrm{F}_{\lambda}(z)\right)^{\prime}}\right)\right\}>-\frac{1}{2} \delta_{1} \eta+\mathrm{p} \eta-(1-\eta) \frac{\beta_{2}}{1-\mathrm{B}^{\prime}}
$$

then $f \in M_{\left[\alpha_{1}\right]}(p ; \alpha ; \beta ; \lambda ; A, B)$ for $\eta \geqslant 0$, where $\delta_{1}=(1-p \beta)\left(\frac{A_{1}-B}{1-B}\right)$.

Proof. Let

$$
h(z)=\frac{\frac{-p}{1-p \beta}\left\{\frac{\mathcal{H}_{p, l, m}\left[\alpha_{1}\right] \mathrm{F}_{\lambda}(z)}{z\left(\mathcal{H}_{p, l, m}\left[\alpha_{1}\right] \mathrm{F}_{\lambda}(z)\right)^{\prime}}+\beta\right\}-\frac{1-\mathrm{A}_{1}}{1-\mathrm{B}}}{1-\frac{1-\mathrm{A}_{1}}{1-\mathrm{B}}} .
$$

Then $h$ is analytic in $\mathbb{U}$. It follows from (3.18) that

$$
\frac{-p \mathcal{H}_{p, l, m}\left[\alpha_{1}\right] F_{\lambda}(z)}{z\left(\mathcal{H}_{p, l, m}\left[\alpha_{1}\right] F_{\lambda}(z)\right)^{\prime}}=\frac{(1-p \beta)\left(A_{1}-B\right) h(z)+\beta_{2}}{1-B},
$$

and

$$
1+\eta \frac{z\left(\mathcal{H}_{p, l, m}\left[\alpha_{1}\right] F_{\lambda}(z)\right)^{\prime \prime}}{\left(\mathcal{H}_{p, l, m}\left[\alpha_{1}\right] F_{\lambda}(z)\right)^{\prime}}=\frac{P+Q h(z)+R z h^{\prime}(z)}{(1-p \beta)\left(A_{1}-B\right) h(z)+\beta_{2}}
$$

where

$$
P=-p \eta(1-B)+(1-\eta)\left[\left(1-A_{1}\right)+p \beta\left(A_{1}-B\right)\right]
$$




$$
Q=(1-p \beta)\left(A_{1}-B\right)(1-\eta), \quad R=-(1-p \beta)\left(A_{1}-B\right) \eta,
$$

combining (3.19) and (3.20), we get

$$
\begin{aligned}
\frac{-p \mathcal{H}_{\mathrm{p}, \mathrm{l}, \mathrm{m}}\left[\alpha_{1}\right] \mathrm{F}_{\lambda}(z)}{z\left(\mathcal{H}_{\mathrm{p}, \mathrm{l}, \mathrm{m}}\left[\alpha_{1}\right] \mathrm{F}_{\lambda}(z)\right)^{\prime}}\left(1+\eta \frac{z\left(\mathcal{H}_{\mathrm{p}, \mathrm{l}, \mathrm{m}}\left[\alpha_{1}\right] \mathrm{F}_{\lambda}(z)\right)^{\prime \prime}}{\left(\mathcal{H}_{\mathrm{p}, \mathrm{l}, \mathrm{m}}\left[\alpha_{1}\right] \mathrm{F}_{\lambda}(z)\right)^{\prime}}\right)= & -\mathrm{p} \eta+(1-\eta) \frac{\beta_{2}}{(1-\mathrm{B})} \\
& +\delta_{1}(1-\eta) \mathrm{h}(z)-\delta_{1} \eta z \mathrm{~h}^{\prime}(z) \\
= & \phi\left(\mathrm{h}(z), z \mathrm{~h}^{\prime}(z) ; z\right),
\end{aligned}
$$

where

$$
\phi(r, s ; t)=-\delta_{1} \eta s+\delta_{1}(1-\eta) r-p \eta+(1-\eta) \frac{\beta_{2}}{1-B} .
$$

Rest of the proof follows by working in similar way as in Theorem 3.4

Theorem 3.6. If $\mathrm{f} \in \Sigma_{\mathrm{p}}$ satisfies anyone of the following conditions:

(i) for $\mathrm{B} \neq-1$,

$$
\left|\left\{\frac{p\left(1-B^{2}\right)}{(1-p \beta)\left(A_{1}-B\right)}\left(\frac{\mathcal{H}_{p, l, m}\left[\alpha_{1}\right] F_{\lambda}(z)}{z\left(\mathcal{H}_{p, l, m}\left[\alpha_{1}\right] F_{\lambda}(z)\right)^{\prime}}+\beta\right)+\frac{1-A_{1} B}{A_{1}-B}\right\}^{\prime}\right| \leqslant \eta|z|^{\tau}
$$

(ii) for $\mathrm{B}=-1, \mathrm{~A}_{1} \neq 1$,

$$
\left|\left(1+\frac{\left(1-A_{1}\right)(1-p \beta)}{p}\left(\frac{z\left(\mathcal{H}_{p, l, m}\left[\alpha_{1}\right] F_{\lambda}(z)\right)^{\prime}}{\mathcal{H}_{p, l, m}\left[\alpha_{1}\right] F_{\lambda}(z)+\beta z\left(\mathcal{H}_{p, l, m}\left[\alpha_{1}\right] F_{\lambda}(z)\right)^{\prime}}\right)\right)^{\prime}\right| \leqslant \eta|z|^{\tau} ;
$$

(iii) for $\mathrm{B}=-1, \mathrm{~A}_{1}=1$,

$$
\left|\left\{\frac{p}{(1-p \beta)}\left(\frac{\mathcal{H}_{p, l, m}\left[\alpha_{1}\right] F_{\lambda}(z)}{z\left(\mathcal{H}_{p, l, m}\left[\alpha_{1}\right] F_{\lambda}(z)\right)^{\prime}}+\beta\right)+1\right\}^{\prime}\right| \leqslant \eta|z|^{\tau}
$$

then $f \in M_{\left[\alpha_{1}\right]}(p ; \alpha ; \beta ; \lambda ; A, B)$, for $0<\eta \leqslant \tau+1$ and $\tau \geqslant 0$.

Proof. (i): If $B \neq-1$, we define the function $\Psi(z)$ by

$$
\Psi(z)=z\left\{\frac{p\left(1-B^{2}\right)}{(1-p \beta)\left(A_{1}-B\right)}\left(\frac{\mathcal{H}_{p, l, m}\left[\alpha_{1}\right] F_{\lambda}(z)}{z\left(\mathcal{H}_{p, l, m}\left[\alpha_{1}\right] F_{\lambda}(z)\right)^{\prime}}+\beta\right)+\frac{1-A_{1} B}{A_{1}-B}\right\},
$$

then $\Psi(z)$ is regular in $\mathbb{U}$ and $\Psi(0)=0$. The condition of theorem gives us that

$$
\left|\left(\frac{\Psi(z)}{z}\right)^{\prime}\right| \leqslant \eta|z|^{\tau}
$$

It follows that

$$
\left|\left(\frac{\Psi(z)}{z}\right)\right|=\left|\int_{0}^{z}\left(\frac{\Psi(t)}{t}\right)^{\prime} d t\right| \leqslant \int_{0}^{|z|} \eta|t|^{\tau} d|t|=\frac{\eta}{\tau+1}|z|^{\tau+1}
$$

This implies that

$$
\left|\left(\frac{\Psi(z)}{z}\right)\right| \leqslant \frac{\eta}{\tau+1}|z|^{\tau+1}<1, \quad(0<\eta \leqslant \tau+1, \quad \tau \geqslant 0) .
$$


Therefore, by the definition of $\Psi(z)$, we conclude that

$$
\left|\frac{p\left(1-B^{2}\right)}{(1-p \beta)\left(A_{1}-B\right)}\left(\frac{\mathcal{H}_{p, l, m}\left[\alpha_{1}\right] F_{\lambda}(z)}{z\left(\mathcal{H}_{p, l, m}\left[\alpha_{1}\right] F_{\lambda}(z)\right)^{\prime}}+\beta\right)+\frac{1-A_{1} B}{A_{1}-B}\right|<1,
$$

which is equivalent to

$$
\left|\frac{p}{(1-p \beta)}\left(\frac{\mathcal{H}_{p, l, m}\left[\alpha_{1}\right] F_{\lambda}(z)}{z\left(\mathcal{H}_{p, l, m}\left[\alpha_{1}\right] F_{\lambda}(z)\right)^{\prime}}+\beta\right)+\frac{1-A_{1} B}{A_{1}-B}\right|<\frac{A_{1}-B}{1-B^{2}} .
$$

Therefore, we conclude that $f(z) \in \mathcal{M}_{\left[\alpha_{1}\right]}(p ; \alpha ; \beta ; \lambda ; A, B)$.

(ii): If $B=-1, A_{1} \neq 1$, we define the function

$$
\Psi(z)=z\left(1+\frac{\left(1-A_{1}\right)(1-p \beta)}{p}\left(\frac{z\left(\mathcal{H}_{p, l, m}\left[\alpha_{1}\right] F_{\lambda}(z)\right)^{\prime}}{\mathcal{H}_{p, l, m}\left[\alpha_{1}\right] F_{\lambda}(z)+\beta z\left(\mathcal{H}_{p, l, m}\left[\alpha_{1}\right] F_{\lambda}(z)\right)^{\prime}}\right)\right),
$$

then $\Psi(z)$ is regular in $\mathbb{U}$ and $\Psi(0)=0$.

Using similar arguments as in proof of (i) and (ii), condition (iii) can be easily verified.

Theorem 3.7. If $f \in \Sigma_{p}$ satisfies

$$
\left|\frac{\frac{1-p \beta}{p} z\left(\mathcal{H}_{p, l, m}\left[\alpha_{1}\right] F_{\lambda}(z)\right)^{\prime}}{\mathcal{H}_{p, l, m}\left[\alpha_{1}\right] F_{\lambda}(z)+\beta z\left(\mathcal{H}_{p, l, m}\left[\alpha_{1}\right] F_{\lambda}(z)\right)^{\prime}}\left(1+M_{p, l, m}^{\alpha_{1}}(F(z))\right)\right|<\frac{A_{1}-B}{1-A_{1}},
$$

then $f \in \mathcal{M}_{\left[\alpha_{1}\right]}(p ; \alpha ; \beta ; \lambda ; A, B)$, for $-1 \leqslant B<A_{1}<\frac{1+B}{2}$, where

$$
M_{p, l, m}^{\alpha_{1}}(F(z))=\frac{z\left(\mathcal{H}_{p, l, m}\left[\alpha_{1}\right] F_{\lambda}(z)\right)^{\prime \prime}}{\left(\mathcal{H}_{p, l, m}\left[\alpha_{1}\right] F_{\lambda}(z)\right)^{\prime}}-\frac{z\left(\mathcal{H}_{p, l, m}\left[\alpha_{1}\right] F_{\lambda}(z)+\beta z\left(\mathcal{H}_{p, l, m}\left[\alpha_{1}\right] F_{\lambda}(z)\right)^{\prime}\right)^{\prime}}{\mathcal{H}_{p, l, m}\left[\alpha_{1}\right] F_{\lambda}(z)+\beta z\left(\mathcal{H}_{p, l, m}\left[\alpha_{1}\right] F_{\lambda}(z)\right)^{\prime}}
$$

Proof. Let

$$
q(z)=\frac{-p}{1-p \beta}\left\{\frac{\mathcal{H}_{p, l, m}\left[\alpha_{1}\right] F_{\lambda}(z)}{z\left(\mathcal{H}_{p, l, m}\left[\alpha_{1}\right] F_{\lambda}(z)\right)^{\prime}}+\beta\right\} .
$$

Then $q(z)$ is analytic in $\mathbb{U}$. The condition of theorem gives us that

$$
\left|z\left(\frac{1}{q(z)}\right)^{\prime}\right|<\frac{A_{1}-B}{1-A_{1}}
$$

that is,

$$
z\left(\frac{1}{q(z)}\right)^{\prime} \prec \frac{A_{1}-B}{1-A_{1}} z .
$$

An application of Lemma 2.3 to (3.22) yields

$$
q(z) \prec \frac{1-A_{1}}{1-A_{1}+\left(A_{1}-B\right) z}=F(z) .
$$

By noting that

$$
\begin{aligned}
\mathfrak{R e}\left(1+\frac{z \mathrm{~F}^{\prime \prime}(z)}{\mathrm{F}^{\prime}(z)}\right) & =\mathfrak{R e}\left(\frac{1-A_{1}-\left(A_{1}-B\right) z}{1-A_{1}+\left(A_{1}-B\right)}\right) \\
& \geqslant \frac{1-A_{1}-\left(A_{1}-B\right)}{1-A_{1}+\left(A_{1}-B\right)} \\
& >0\left(-1 \leqslant B<A_{1}<\frac{1+B}{2}\right),
\end{aligned}
$$

which implies that the region $F(\mathbb{U})$ is symmetric with respect to the real axis and $F$ is convex univalent in 
$\mathbb{U}$. Therefore, we have

$$
\mathfrak{R e}(F(z))>F(1)=\frac{1-A_{1}}{1-B} .
$$

Combining (3.21), (3.23) and (3.24), we deduce that for $\left(-1 \leqslant B<A_{1}<\frac{1+B}{2}\right)$,

$$
\mathfrak{R e}\left\{\frac{p}{1-p \beta}\left\{\frac{\mathcal{H}_{p, l, m}\left[\alpha_{1}\right] F_{\lambda}(z)}{z\left(\mathcal{H}_{p, l, m}\left[\alpha_{1}\right] F_{\lambda}(z)\right)^{\prime}}+\beta\right\}\right\}<-\frac{1-A_{1}}{1-B}
$$

which is equivalent to

$$
\frac{p}{1-p \beta}\left\{\frac{\mathcal{H}_{p, l, m}\left[\alpha_{1}\right] F_{\lambda}(z)}{z\left(\mathcal{H}_{p, l, m}\left[\alpha_{1}\right] F_{\lambda}(z)\right)^{\prime}}+\beta\right\} \prec-\frac{1+A_{1} z}{1+B z} .
$$

This evidently completes the proof of Theorem 3.7.

\section{Acknowledgment}

The work here is supported by MOHE grant: FRGS/1/2016/STG06/UKM/01/1.

\section{References}

[1] N. E. Cho, I. H. Kim, Inclusion properties of certain classes of meromorphic functions associated with the generalized hypergeometric function, Appl. Math. Comput., 187 (2007), 115-121. 1

[2] P. L. Duren, Univalent functions, Grundlehren der Mathematischen Wissenschaften [Fundamental Principles of Mathematical Sciences], Springer-Verlag, New York, (1983). 1

[3] J. Dziok, H. M. Srivastava, Classes of analytic functions associated with the generalized hypergeometric function, Appl. Math. Comput., 103 (1999), 1-13. 1

[4] I. S. Jack, Functions starlike and convex of order $\alpha$, J. London Math. Soc., 3 (1971), 469-474. 2.1

[5] W. Janowski, Some extremal problems for certain families of analytic functions, I, Bull. Acad. Polon. Sci. Sr. Sci. Math. Astronom. Phys., 21 (1973), 17-25. 1

[6] J.-L. Liu, A linear operator and its applications on meromorphic p-valent functions, Bull. Inst. Math. Acad. Sinica, 31 (2003), 23-32. 1

[7] J.-L. Liu, H. M. Srivastava, A linear operator and associated families of meromorphically multivalent functions, J. Math. Anal. Appl., 259 (2001), 566-581. 1

[8] J.-L. Liu, H. M. Srivastava, Classes of meromorphically multivalent functions associated with the generalized hypergeometric function, Math. Comput. Modelling, 39 (2004), 21-34. 1

[9] J.-L. Liu, H. M. Srivastava, Subclasses of meromorphically multivalent functions associated with a certain linear operator, Math. Comput. Modelling, 39 (2004), 35-44. 1

[10] L.-N. Ma, S.-H. Li, On certain class of meromorphically multivalent reciprocal starlike functions associated with the LiuSrivastava operator defined by subordination, Filomat, 28 (2014), 1965-1982. 1

[11] S. S. Miller, P. T. Mocanu, Differential subordinations and inequalities in the complex plane, J. Differential Equations, 67 (1987), 199-211. 2.2

[12] S. S. Miller, P. T. Mocanu, Differential subordinations, Theory and applications, Monographs and Textbooks in Pure and Applied Mathematics, Marcel Dekker, Inc., New York, (2000). 1

[13] R. K. Raina, H. M. Srivastava, A new class of meromorphically multivalent functions with applications to generalized hypergeometric functions, Math. Comput. Modelling, 43 (2006), 350-356. 1

[14] H. Silverman, E. M. Silvia, Subclasses of starlike functions subordinate to convex functions, Canad. J. Math., 37 (1985), 48-61. 1

[15] H. M. Srivastava, S. Owa (ed.), Current topics in analytic function theory, World Scientific Publishing Co., Inc., River Edge, NJ, (1992). 1

[16] H. M. Srivastava, J. Patel, Some subclasses of multivalent functions involving a certain linear operator, J. Math. Anal. Appl., 310 (2005), 209-228. 1

[17] Y. Sun, W.-P. Kuang, Z.-G. Wang, On meromorphic starlike functions of reciprocal order $\alpha$, Bull. Malays. Math. Sci. Soc., 35 (2012), 469-477. 1, 1

[18] B. A. Uralegaddi, C. Somanatha, New criteria for meromorphic starlike univalent functions, Bull. Austral. Math. Soc., 43 (1991), 137-140. 1

[19] D.-G. Yang, On a class of meromorphic starlike multivalent functions, Bull. Inst. Math. Acad. Sinica, 24 (1996), 151-157. 1

[20] D.-G. Yang, Some criteria for multivalently starlikeness, Southeast Asian Bull. Math., 24 (2000), 491-497. 1, 2.3 